\title{
Expression of Math1 and HES5 in the Cochleae of Wildtype and Jag2 Mutant Mice
}

\author{
Pamela J. Lanford, ${ }^{1 *}$ Ranu Shailam, Christine R. Norton ${ }^{2}$ Thomas Gridley, and \\ MATTHEW W. KeLLEY \\ ${ }^{1}$ Department of Cell Biology, Georgetown University School of M edicine, Washington, DC 20007 USA \\ ${ }^{2}$ T he Jackson Laboratory, Bar H arbor, M E 04609 USA
}

Received: 12 May 2000; Accepted: 25 May 2000; O nline publication: 15 August 2000

\section{ABSTRACT}

The sensory epithelium within the mammalian cochlea (the organ of Corti) is a strictly ordered cellular array consisting of sensory hair cells and nonsensory supporting cells. Previous research has demonstrated that Notch-mediated lateral inhibition plays a key role in the determination of cell types within this array. Specifically, genetic deletion of the Notch ligand, Jagged2, results in a significant increase in the number of hair cells that develop within the sensory epithelium, presumably as a result of a decrease in Notch activation. In contrast, the downstream mediators and targets of the Notch pathway in the inner ear have not been determined but they may include genes encoding the proneural gene $M$ ath 1 as well as the HES family of inhibitory bHLH proteins. To determine the potential roles of these genes in cochlear development, in situ hybridization for $M$ ath1 and HES5 was performed on the cochleae of wildtype vs. Jagged2 mutants (Jag2 ${ }^{\triangle \mathrm{DSL}}$ ). Results in wild-type cochleae show that expression of $M$ ath1 transcripts in the duct begins on E13 and ultimately becomes restricted to hair cells in the sensory epithelium. In contrast, expression of HES5 begins on E15 and becomes restricted to supporting cells in the epithelium. Results in Jag2 mutant cochleae suggest that $M$ ath1 transcripts are ultimately maintained in a larger

*Current address: National Institute on Deafness and O ther Communication Disorders, The National Institutes of Health, 5 Research Court, Rockville, MD 20850.

Correspondence to: Dr. Pamela Lanford - NIDCD/ NIH • 5 Research Court - Rockville, MD 20850 USA. Telephone: (301) 435-8074; fax (301) 496-0190; e-mail: lanfordp@nidcd.nih.gov number of cells as compared with wildtype, while transcripts for HES5 are dramatically reduced throughout the epithelium. These results are consistent with the hypothesis that activation of Notch via Jagged 2 acts to inhibit expression of $M$ ath 1 in cochlear progenitor cells, possibly through the activity of HES5.

Keywords: Notch, Jagged2, lateral inhibition, inner ear, hair cell

\section{INTRO DUCTION}

The restriction of multipotent embryonic progenitor cells to specific cell fates is a complex and highly regulated developmental process. The molecular mechanisms that drive this process have been characterized most successfully in invertebrate model systems where structure and development are strictly ordered and perturbation sof the system are readily detectable (e.g., Drosophila retina) (reviewed in Wolff and Ready 1993). In vertebrate species, similar highly structured systems are rare. However, the sensory epithelium within the mammalian cochlea (the organ of Corti) is one example of a vertebrate system in which the physical arrangement of cell types and the spatiotemporal sequence of their development are highly invariant. The organ of Corti contains four rows of mechanosensory hair cells, including a single row of inner hair cells and three rows of outer hair cells. Within each row, hair cells are separated from one another by an interceding nonsensory supporting cell, forming a mosaic that extends along the length of the cochlear duct (reviewed in Lim and Rueda 1992). During embryogenesis, the development of this mosaic begins 
near the base of the cochlea and proceeds toward the apex in a wave of differentiation ( Lim and Anniko 1985) that is reminiscent of development in the compound eye of Drosophila (Wolff and Ready 1993).

The results of previous studies have led to the suggestion that cell fates within the developing organ of Corti may be determined in part through intercellular inhibitory interactions (lateral inhibition) (Corwin et al. 1991; Lewis 1991). In particular, Iaser microbeam ablation of individual differentiating hair cells within the embryonic cochlea demonstrated that removal of these cells al lows adjacent progenitor cells to alter their fates and to develop as replacement hair cells (Kelley et al. 1995). This result suggests that newly developing hair cells produce an inhibitory signal that prevents the differentiation of the hair cell phenotype in their immediate neighbors.

Similar lateral inhibitory signals play a role in the determination of cell types in a variety of invertebrates and nonplacental vertebrates ( reviewed in Muskavitch 1994; Lewis 1996). In addition, these signals appear to be one component of a larger series of determining steps that progressively restricts the number of progenitor cells that may become competent to develop as a given cell type (reviewed in Campos-Ortega 1995; Modollel 1997). Specifically, lateral inhibition diverts a subset of cells within the progenitor pool from advancing from one level of competency to the next by repressing the activity of a default developmental program (Dorsky et al. 1997). The subset of cells that are diverted by lateral inhibition are subsequently returned to the progenitor pool.

This mechanism plays a key role in the determination of cell fates in the developing nervous system in both vertebrates and invertebrates (reviewed in Anderson and Jan 1997). For example, during development of Drosophila, neural competency is established in groups of progenitor cells through the activity of "proneural" basic helix-loop-helix (bHLH) genes such as atonal (ato) and the achaetescute (AS-C) complex (Jarmen et al. 1993, 1994; Modellel 1997). All progenitors that express these proneural genes have the potential to develop as neural cell types and, therefore, constitute a proneural "equivalence group" (Doe and Goodman 1985). As development proceeds, a subset of cells within this equivalence group is diverted from the neural fate through lateral inhibition, mediated through the activity of "neurogenic" genes such as $\mathrm{N}$ otch, Delta, and the en han cer of split complex [E(spl) ] (reviewed in Artavanis-Tsakonis et al. 1995). Binding of the receptor, Notch, to its ligand, Delta, results in the activation of a downstream pathway that results in the increased expression of $E(s p l)$ (reviewed in Bray 1997). The bHLH proteins encoded by $E(s p l)$ negatively regulate the expression of neural phenotypes through repression of proneural genes (Fig. 1) ( $N$ akao

\section{The Notch Signaling Pathway}

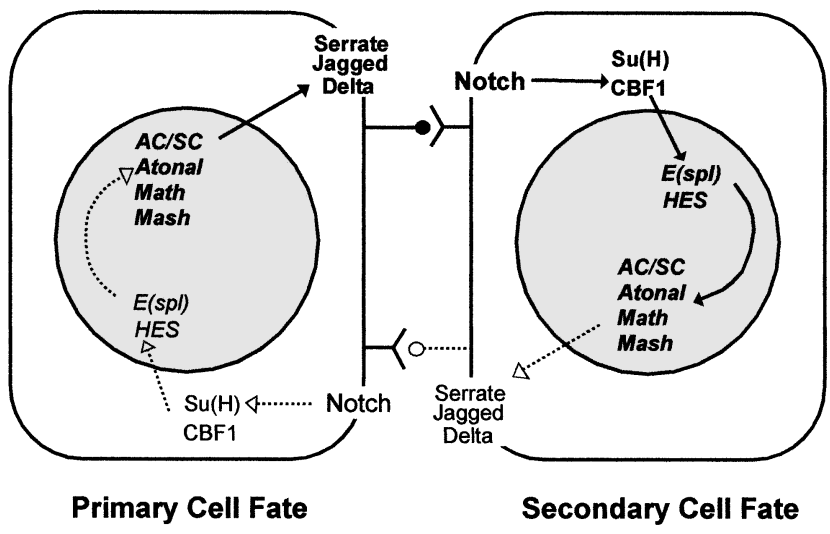

FIG. 1. Diagram of the $\mathrm{N}$ otch pathway. Research in vertebrates and invertebrates indicates that the N otch signaling pathway may function in the following manner: During embryonic development, a population of progenitor cells expresses the Notch receptor $(\mathrm{N})$. Within this progenitor pool, specific cells begin to express proneural genes (atonal, acheate-scute, Math, and MASH ). O ne effect of proneural gene expression is the upregulation of genes encoding the $\mathrm{N}$ otch ligands (D elta, Serrate, and Jagged). In addition, a single progenitor cell may produce more of the ligand than its neighbors, via random fluctuations in gene expression or through some additional extrinsic or intrinsic biasing mechanism. Increased levels of ligand expression in a given progenitor result in a subsequent increase in the activation of $\mathrm{N}$ otch in adjacent cells. Increased activation of $\mathrm{N}$ otch in turn results in the upregulation of downstream effector molecules (enhancer of split, $\mathrm{HES}$ ) that actas negative regulators of proneural gene expression. Ultimately, the regulatory feeback loop between proneural gene expression and activation of the $\mathrm{N}$ otch pathway leads to the establishment of two stable populations of progenitor cells. O ne population continues to express proneural genes and the $\mathrm{N}$ otch ligand and will develop as the primary cell type. The second population does not express proneural genes and the Notch ligand and will develop as a secondary or tertiary cell type.

and Campos-Ortega 1996; Giebel and CamposOrtega 1997; Jimenez and Ish-Horowicz 1997; Bray 1997).

Mammalian homologs for many of the neurogenic and proneural genes have now been identified, including HES [E(spl)], Mash (AS-C), and M ath (ato) (Akazawa et al. 1992, 1995; Sasai et al. 1992; Sommer et al. 1996; $M a$ et al. 1996). Recent studies have demonstrated that activation of Notch results in the subsequent activation of specific HES genes (HES1 and HES5) (Tomita et al. 1996; Kageyama and Nakanishi 1997; O htsuka et al. 1999). In addition, mutations in these and other Notch pathway-related genes results in the upregulation of mammalian proneural genes and neural markers (Ishibashi et al. 1995; Pompa et al. 1997; O htsuka et al. 1999). Until recently, however, a role for any of the neurogenic genes in lateral inhibition had not been demonstrated directly in mammals. Studies from several laboratories have indi- 
cated that the Notch pathway is involved in the development of the vertebrate inner ear (Lindsell et al. 1996; Luo et al. 1997; Adam et al. 1998; Haddon et al. 1998; Lanford et al. 1999; Morrison et al. 1999). In the mammalian cochlea, Notch1 and its ligands, encoded by Jag2 and Delta1 (DII1), are expressed in a manner consistent with a role in lateral inhibition ( Lanford et al. 1999; M orrison et al. 1999). In addition, cochleae from embryonic mice containing a targeted deletion of the Jag2 gene ( Jag2 ${ }^{\mathrm{DDSL}}$ ) (Jiang et al. 1998) contain a greater number of hair cells in the sensory epithelium compared with wildtype (Lanford et al. 1999). Combined, these results support a role for Notch-mediated lateral inhibition in the determination of the number of progenitor cells that will develop as hair cells.

The specific genes that are regulated by activation of the Notch pathway and the downstream molecules that mediate this regulation have not yet been determined in the inner ear; however, the results of a recent study indicate that the proneural gene $M$ ath1 may be one candidate. Bermingham and colleagues (1999) demonstrated the presence of $\mathrm{M}$ ath1 promoter activity in the developing organ of Corti at developmental time points consistent with the determination of sensory cell fates. Their study also demonstrated that genetic deletion of $M$ ath1 results in the complete absence of hair cells in the sensory epithelium. While a specific regulatory relationship between the Notch pathway and the expression of $M$ ath1 has not been demonstrated in the inner ear, the results of previous studies indicate that the activity of mammalian proneural proteins can be repressed by HES proteins (Sasai et al. 1992; Akazawa et al. 1995). Consequently, it seems possible that Notch-dependent bH LH genes such as HES5 are expressed in the developing cochlea and that these genes are responsible for the diversion of sensory progenitor cells from the hair cell fate, via repression of $M$ ath1. The first aim of the present study was to confirm the expression pattern of $M$ ath 1 in the developing organ of Corti and to determine whether HES5 is expressed in the cochlear duct in a manner consistent with the activation of Notch. The second aim of the study was to determine whether the deletion of Jag2 alters the expression patterns of these genes in a manner consistent with a decrease in Notch activation. Specifically, we hypothesized that the Notch pathway inhibits sensory progenitor cells from differentiating as hair cells through the activation of $\mathrm{HES}$ genes and the subsequent repression of $M$ ath1. Deletion of Jag2 should result in a decrease in expression of HES genes and an increase in the number of cells that maintain Math1 expression and differentiate as hair cells.

\section{MATERIALS AND METHODS}

In situ hybridization

Timed-mated pregnant ICR mice were euthanized with $\mathrm{CO}_{2}$ and embryos were obtained on gestational days 12-18 (E12-18) or postnatal days $0-3$ ( P0-3) (staging according to Kaufman, 1992). The temporal bones were dissected out of the cranium, and an opening was made into the cochlear region to ensure adequate fixation. Tissues were fixed in $4 \%$ paraformaldehyde, dehydrated to $100 \%$ methanol, and stored at $-20^{\circ} \mathrm{C}$. Prior to processing for in situ hybridization, the cochleae were rehydrated and the cochlear capsule and roof of the cochlear duct were removed to expose the developing sensory epithelium.

Synthesis of digoxygenin-labeled cRNA probes was performed according to Wilkinson and Nieto (1993). RNA probes were synthesized from cDNAs for HES5 (Akazawa et al. 1992) and M ath1 (Akazawa et al. 1995). In situ hybridization procedures were modified from Wilkinson and Nieto (1993). Briefly, cochleae were rinsed in phosphate buffered saline (PBS) plus $0.1 \%$ Tween 20 , treated with $6 \% \mathrm{H}_{2} \mathrm{O}_{2}$, and digested in Proteinase $\mathrm{K}$. The tissues were incubated in prehybridization solution for 2 hours at $70^{\circ} \mathrm{C}$, then exposed to RNA probes overnight at $70^{\circ} \mathrm{C}$. Bound probes were detected via a standard alkaline phosphatase immunohistochemical reaction. A minimum of two cochleae were processed per time point per probe. Selected tissues were embedded in Tissue-Tek O.C.T. embedding compound and sectioned at 8-10 $\mu \mathrm{m}$.

In situ hybridization and morphological analyses of cochleae from Jag2 mutant mice

Animals homozygous for a targeted deletion of Jag2 ( referred to in this text as "Jag2 mutants") die at birth as a result of craniofacial defects that are not related to the development of the ear (Jiang et al. 1998). Therefore, embryos were obtained at developmental time points between E14.5 and E17.5 as described above. Genotypes were determined initially on the basis of morphological characteristics and subsequently confirmed by polymerase chain reaction (PCR) (Jiang et al. 1998). Cochleae were dissected, fixed, and prepared for in situ hybridization as described. Mutant cochleae were processed with probes against $M$ ath 1 and $H E S 5$, as well as Brn3.1 (kindly provided by E. Huang and L. Reichardt), an early marker for hair cell differentiation (Xiang, et al. 1998). Control cochleae (ICR strain for Brn 3.1 cochleae; Jag2 wildtype for M ath 1 and H ES5) were also processed for in situ hybridization.

To examine the morphological effects of deletion 
of Jag2, cochleae were fixed in gluteraldehyde, dehydrated, and embedded in methacrylate (I mmunobed, Polysciences, Inc., Warrington, PA). Tissues were sectioned at 3-5 $\mu \mathrm{m}$ on a rotary microtome, mounted on glass slides, and stained with thionine.

\section{RESU LTS}

The results of previous studies in the mammalian cochlea suggest that the determination of hair cell fates in the organ of Corti begins in the base of the cochlea at about E13 and extends along the length of the cochlea as development progresses. Early markers of hair cell differentiation such as the Notch ligands Jag2 and Delta1, as well as M yoVI, MyoVIIA and Brn3.1, are detected in the base of the cochlea beginning at E13 and extend to the apex of the cochlea by the time of birth ( $\mathrm{H}$ asson et al. 1995, 1997; Erkman et al. 1996; Xiang et al. 1997; Lanford et al. 1999; Morrison et al. 1999). In addition, the morphological characteristics of hair cells (e.g., stereociliary bundles) first become identifiable in the base of the cochlea at about E15, but not in the apical regions of the epithelium until late gestational or early postnatal time points ( $\mathrm{Lim}$ and Anniko 1985; Lim and Rueda 1992). Based on these results, the developmental time frame between E13 and E17 appears to be critical for the determination of cell fates al ong the length of the organ of Corti. Consequently, in situ hybridization for proneural and neurogenic gene expression in the cochlear duct was performed at time points that bracket this critical developmental period (E12-P3).

\section{Expression of Math1 in the developing cochlea}

The expression pattern of $M$ ath1 mRNA in the developing organ of Corti is consistent with the results of a recent study demonstrating promoter activity for this gene in the developing sensory epithelium (Bermingham et al. 1999) (Fig. 2). Transcripts for M ath1 can first be detected in the basal turn of the cochlea beginning on E13 in a relatively narrow band of cells (Fig. $2 \mathrm{~A}-\mathrm{C}$ ). Examination of sectioned cochleae indicates that the location of this band correlates with the region of the cochlear duct that will develop as the sensory epithelium. In addition, the band of $M$ ath 1 expression at E13 spans the thickness of the ventral floor of the duct, from the basement membrane to the lumenal surface (Fig. 2B,C).

By $E 15$, the band of $M$ ath1 expression appears to have broadened slightly and has extended to nearly the full length of the cochlear duct (Fig. 2D). Tissue sections demonstrate that $M$ ath 1 transcripts are restricted to the region of the cochlear duct overlying the spiral vessel, a transient structure that serves as a landmark for the developing sensory epithelium (Fig. $2 \mathrm{E})$. While the expression of $M$ ath 1 still spansthe thickness of the membrane at this time point, transcripts appear to be becoming restricted to cells in the lumenal half of the epithelium (Fig. 2E,F). By E17, M ath1 transcripts are clearly restricted to inner and outer hair cells along the full length of the sensory epithelium (Fig. 2G-I). Qualitative assessment of Math1 expression in tissue sections suggests that the number of cells that express this proneural gene is reduced at E17.5 by comparison with a comparable region of the duct at earlier developmental time points. Finally, by P3 Math1 expression begins to decrease in the base of the cochlea but remains expressed in the apex, suggesting that this gene is expressed transiently within the cochlear duct and that both upregulation and downregulation of $M$ ath1 occur in basal-to-apical gradients (not shown).

\section{Expression of HES5 in the developing cochlea}

Initiation of H ES5 expression beginsat E15 in a narrow band of cells originating in the base of the cochlea and extending toward the apex (Fig. 3A). At this time point, the band of $\mathrm{HES5}$ expression spansthe thickness of the ventral floor of the duct (Fig. 3B,C) and is located in the region of the duct overlying the spiral vessel (Fig. 3B,C). In addition, the band of HES5 expression appears to occur within the somewhat broader domain of $M$ ath 1 expression, based on the positions of the expression bands relative to the spiral vessel ( compare Figs. 2E, F and $3 \mathrm{~B}, \mathrm{C}$ ). At E 16, the band of HES5 expression has expanded in both the neural and abneural directions and along the basal-to-apical axis (Fig. 3D-F). Transcripts for HES5 appear to be more intense in cells adjacent to the basement membrane (Fig. 3E,F). In addition, the band of HES5 expression appears to become divided into two distinct regions by interceding H ES5-negative cells. This division begins in the base of the cochlea and isstill evident in the apical region of the epithelium at E17 (Fig. 3G) . Sections through the apical turn of an E17 cochlea indicate that, between E15 and E17, the domain of H ES5 expression includes both supporting cells within the sensory epithelium and cells in the inner sulcus region (Fig. $3 \mathrm{H}$ ). As development continues, the expression of HES5 transcripts becomes progressively downregulated from the inner sulcus region of the duct through the sensory epithelium. This downregulation is demonstrated in sections from the basal turn of an E17 cochlea, which indicates expression of H ES5 restricted to Dieter's cells located in the abneural half of the sensory epithelium (Fig. $3 \mathrm{l}, \mathrm{J}$ ). By P0, HES5 expression is absent in the basal turn of the cochlea but persists in the apical turns, suggesting that tran- 
A

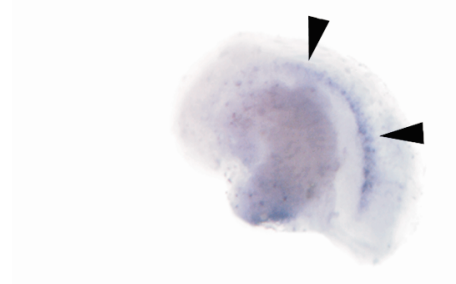

D

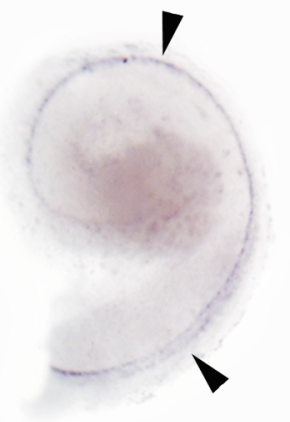

E

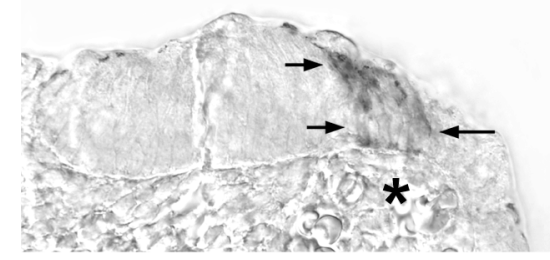

$\mathbf{F}$

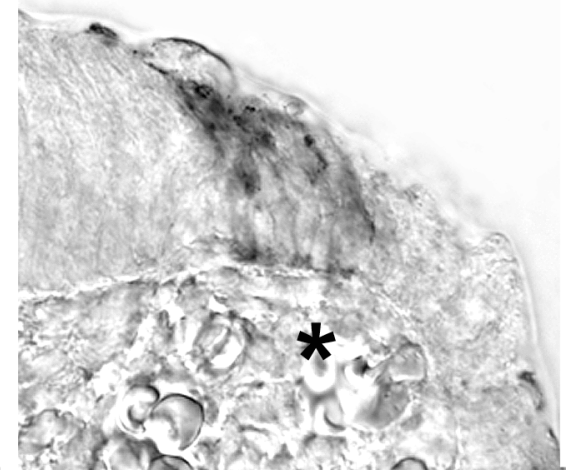

G

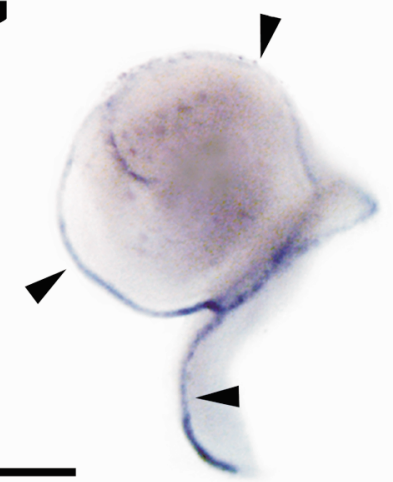

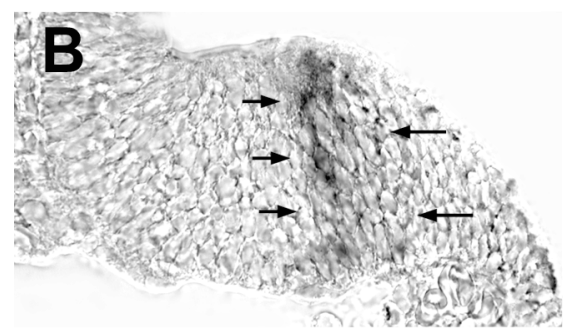
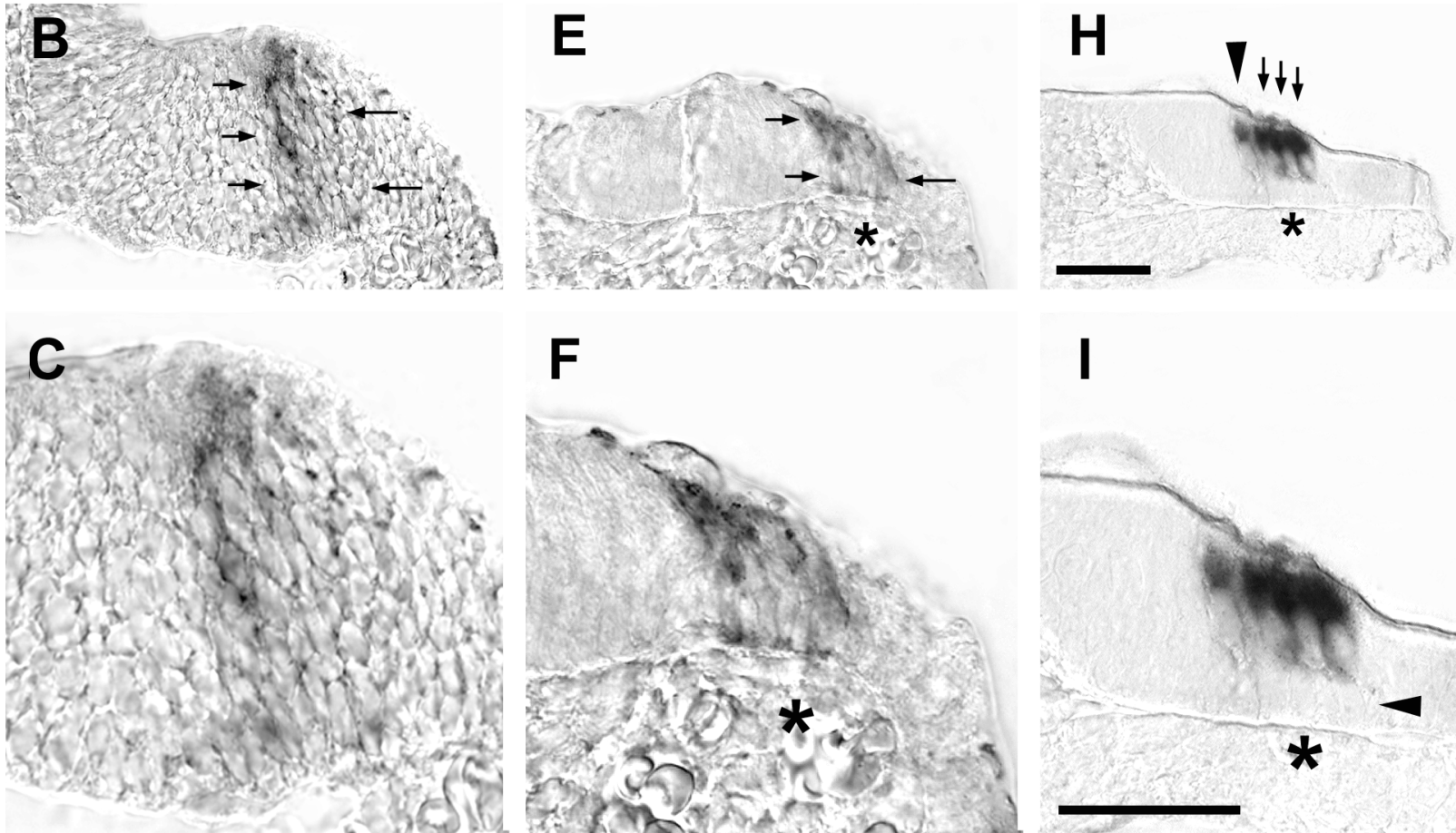

I

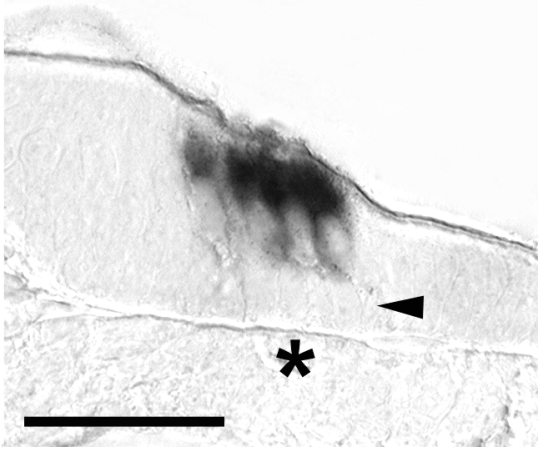

FIG. 2. In situ hybridization for Math1 in cochleae from E13, E15, and E17. The roof of the cochlear duct has been removed. A. Whole mount of the cochlear duct at E13. Math1 transcripts are expressed in a relatively narrow band of cells that does not yet extend the full length of the epithelium (arrowheads). B. Cryosection through an E13 cochlea demonstrates that the expression domain for Math 1 extends from the lumenal to basal surfaces (arrows). C. Higher magnification view of $B$. Individual cells expressing $M$ ath 1 are clearly labeled at multiple levels within the thickness of the duct. $\mathbf{D}$. By E15, the Math1 expression domain has expanded along the full length of the duct. E. Cryosection from the middle turn of the cochlea demonstrates $M$ ath1 expression in cells located throughout the region of the duct that will develop as the sensory epithelium (arrows; asterisk indicates

scription of this gene is progressively downregulated in a basal-to-apical gradient (not shown).

\section{Morphology and gene expression patterns within the ears of Jag2 mutant mice}

The results of a recent study have demonstrated that targeted deletion of the N otch ligand, Jagged2, results

position of the spiral vessel). F. Higher magnification of E. Math1 expression appears to be more intense in cells located in the lumenal half of the epithelium (asterisk indicates spiral vessel). G. At E17, transcripts for $M$ ath 1 persist along the entire length of the cochlear duct (arrowheads). Scale bar equals $250 \mu \mathrm{m}$ (same in A and D). $\mathbf{H}$. Sections through the base of the cochlea demonstrate that expression of $M$ ath 1 is restricted to developing hair cells (arrowhead indicates inner hair cell, arrows indicate outer hair cells, asterisk indicates the spiral vessel). Scale bar equals $25 \mu \mathrm{m}$ (same in B and E). I. Higher magnification of $\mathrm{H}$. Nuclei of Deiter's cells can be distinguished in this section (asterisk indicates the spiral vessel). Expression continues through P3, but appears to become downregulated in a basal-toapical gradient (not shown). Scale bar equals $25 \mu \mathrm{m}$ (same in C and F).

in a significant increase in the number of cells that differentiate as hair cells in the organ of Corti (Lanford et al. 1999). Presumably, this is because of a decrease in the levels of Notch activation, which allows more progenitor cells to become committed to the hair cell fate. Since mutant pups die at birth, our previous study examined cochleae from Jag2 mutant animals at the latest possible embryonic time point 
A

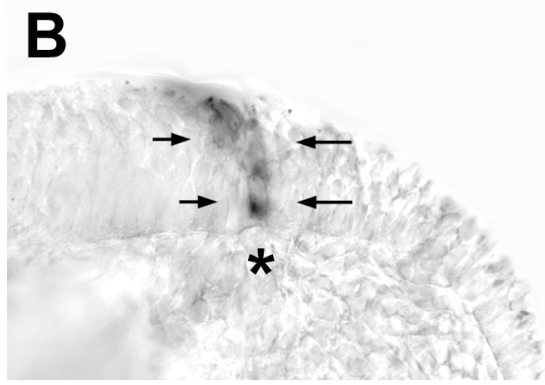

C

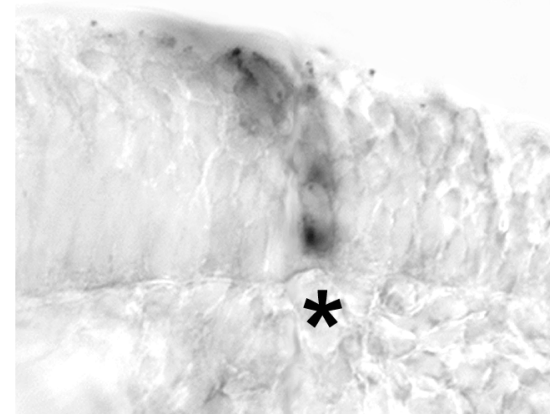

FIG. 3. In situ hybridization for HES5 in cochleae from E15, E16, and E17. The roof of the cochlear duct has been removed. A. At E15, HES5 expression begins in the cochlear duct in a highly restricted band of cells that extends from the base of the cochlea toward its apex (arrowhead). B. Cryosections of E15 cochleae demonstrate that this expression is located in the developing sensory region of the duct (arrows; asterisk indicates position of the spiral vessel). This image is a composite of two photographs of the same section, taken at slightly different planes of focus. C. Higher magnification of B. N ote that the width of HES5 expression is relatively narrow compared with the expression band of $M$ ath 1 at the same time point (see Fig2E,F) (asterisk indicates spiral vessel). D. As development proceeds through E16, expression of HES5 extends along the basal-to-apical axis of the duct (arrowheads). E. The band of HES5 expression has broadened relative to its width atE15 (arrows; asterisk indicates the spiral vessel). F. Higher magnification of E. HES5 expression appears to be more intense in cells located in the basal half of the epithelium (asterisk indicates the spiral vessel). G. At E17, expression of HES5 extends along the entire length of the epithelium (arrowheads). As HES5
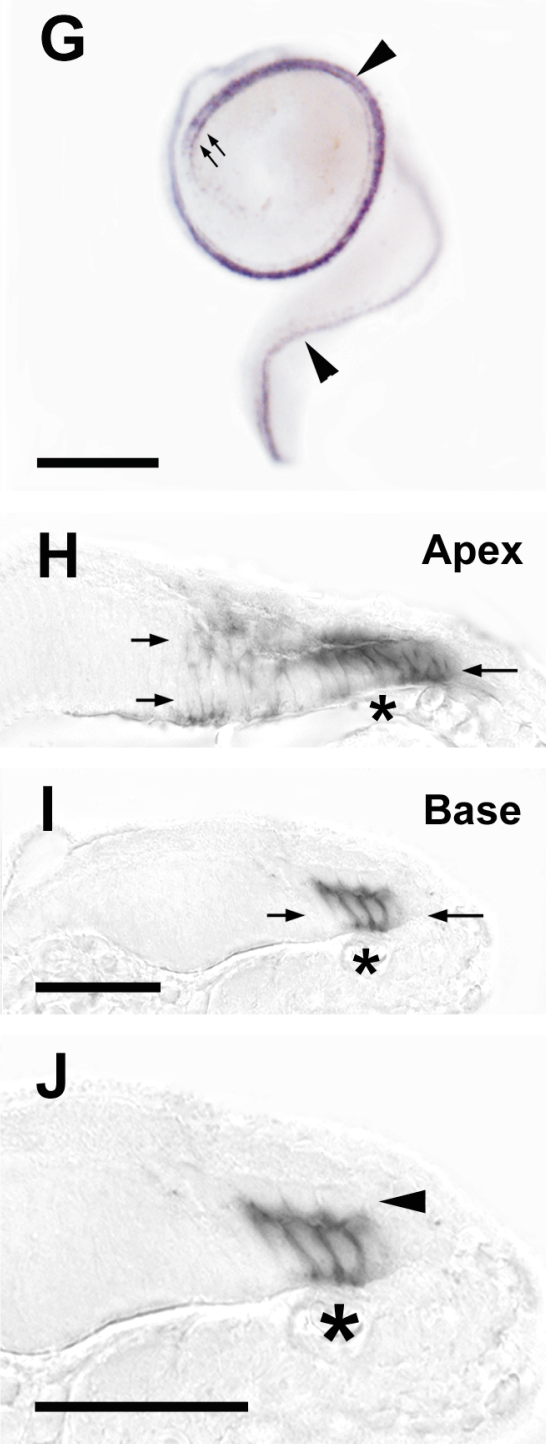

transcripts are expressed along the cochlear duct, subdivisions within the band of expression are distinguishable. These subdivisions are still clearly visible in the apex of the cochlea at E17 (double arrows) Scale bar equals $250 \mu \mathrm{m}$ (same in A and D). H. Cryosection through the apical turn of the E17 cochlea shows the relatively broad band of HES5 expression that appears to include inner phalangeal cells as well as developing Deiter's cells (arrows; asterisks indicates spiral vessel). This section is slightly oblique. I. In contrast to the apical section, a section through the base of the E17 cochlea shows HES5 expression restricted to Deiter's cells located in the abneural region of the epithelium (arrows; asterisk indicates spiral vessel). This result suggests that HES5 expression is progressively downregulated from the neural side of the developing epithelium to the abneural side. Scale bar equals $25 \mu \mathrm{m}$ (same in B, E, H). J. Higher magnification of I. N uclei of hair cells can be distinguished in this section (asterisk indicates the spiral vessel). As development continues, expression of HES5 appears to become completely downregulated in an apicalto-basal gradient, but persists in the apex of the cochlea through at least P5 (not shown). Scale bar equals $25 \mu \mathrm{m}$ (same in C and F).

since hair cells in this region of the epithelium are still not fully differentiated at E17 and could not be reliably counted. In the present study, we have specifi-
(E17), to ensure the greatest degree of cellular differentiation within the epithelium. The apical one-third of the cochlear duct was omitted from the analysis, 
cally examined sections from both the apical and basal regions of E17 mutant cochleae in order to compare the morphology of the duct at early versus late points in hair cell development. Results in Jag2 mutant cochleae indicate that additional developing hair cells are present along the length of the epithelium at the earliest points at which these cells become morphologically identifiable (Fig. 4A-D). The identity of these additional cells was confirmed by expression of an early marker of hair cell differentiation, Brn 3.1 (Fig. 4E,F). These results support the hypothesis that Notch signaling acts at an early time point to regulate the number of cells that differentiate as hair cells. The results also suggest that the number of cells within the initial pool of sensory progenitor cells is larger number than is required to form the final population of hair cells in the epithelium.

\section{Expression of Math1 and HES5 in Jag2 mutant cochleae}

Results from normal cochleae (see above) indicate that transcripts for $M$ ath1 are expressed in the cochlea at least one day prior to the onset of Jag2 expression ( $M$ ath1 at E13, Jag2 at E14.5). Consequently, the initiation of $M$ ath 1 expression appears to be independent of Jag2-dependent Notch signaling. Results from Jag2 mutant cochleae indicate, however, that the continued expression of $M$ ath 1 in sensory progenitor cells is regulated by the Notch pathway. In Jag2 mutant cochleae, $M$ ath1 transcripts were consistently detected in the additional inner hair cells present in the epithelium (Fig. 4G,H). O ur previous research has demonstrated that the deletion of Jag2 results in a statistically significant increase in the number of inner hair cells (and total hair cells) that develop in the organ of Corti (Lanford et al. 1999). Since M ath1 expression is maintained only in cells that differentiate as hair cells, the number of $M$ ath1-positive cells that are ultimately present in Jag2 mutant cochleae must be greater than in wildtype.

In contrast to $M$ ath 1 , both the initiation and maintenance of HES5 transcripts is dramatically altered in cochleae from Jag2 mutants. In normal cochleae, transcripts for HES5 are detectable in the developing sensory epithelium by E15 and continue to be expressed through at least P0 (see above). By comparison, the initial expression of HES5 in cochleae from Jag2 mutant animals is markedly decreased, and the overall level of HES5 expression remains low or undetectable through E17.5 (Fig. 5A-D). A similar downregulation of HES5 was also present in the sensory epithelia of the semicircular canals (canal cristae) which also express HES5 (Shailam et al. 1999). These results are consistent with the role of HES5 as a downstream mediator of Notch signaling and support the hypothesis that
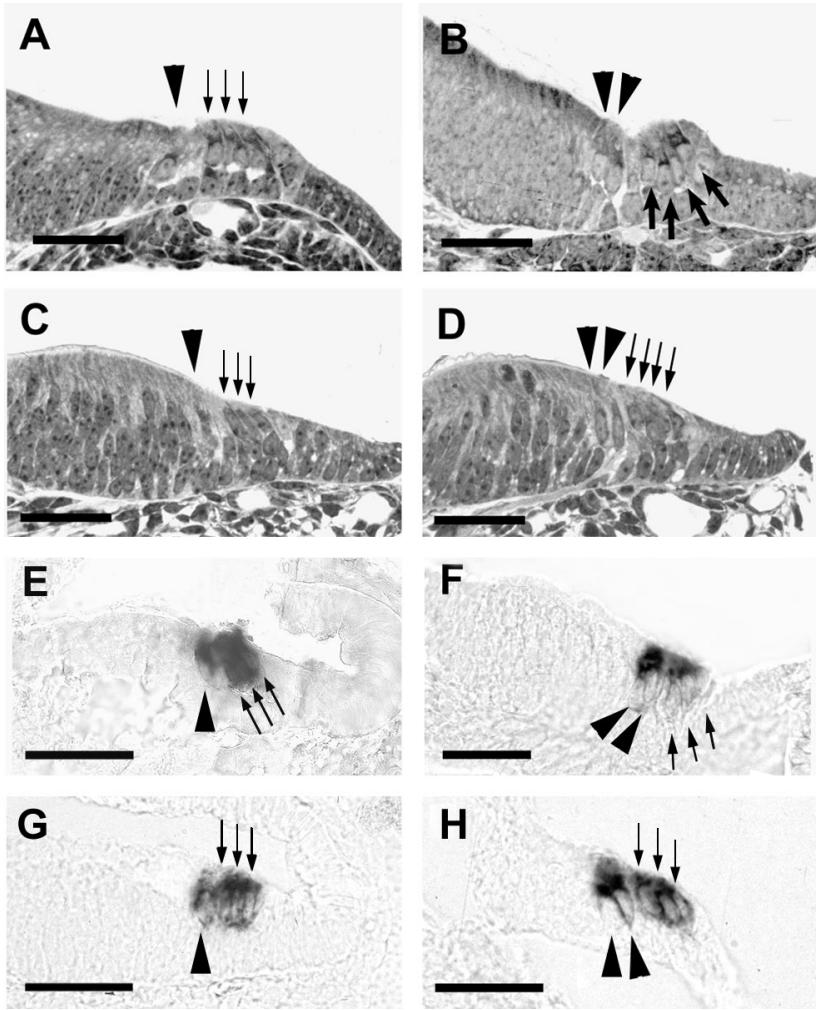

FIG . 4. Effects of deletion of Jag2 on the development of hair cells in the organ of Corti. A-D. M orphological analysis of the apex versus the base of cochlea from E17.5 mutants demonstrates that additional developing hair cells are present throughout the epithelium at the earliest developmental time points that these cells can be identified. A. Cross section through the basal turn of the cochlear duct from an E17.5 wildtype animal. A single inner hair cell (arrowhead) and three outer hair cells (arrows) are present within the developing organ of Corti. B. Cross section through the basal turn of the cochlear duct from an E17.5 Jag2 mutant. Two inner hair cells (arrowheads) and four outer hair cells (arrows) are present. C. Cross section through the apical turn of the cochlear duct from an E17.5 wildtype animal. At this stage in development, the organ of Corti in the apical turn is still relatively undifferentiated. However, the precursors of a single inner hair cell (arrowhead) and three outer hair cells (arrows) can be identified within the ventral epithelium. D. Cross section through the apical turn of the cochlear duct from an E17.5 Jag2 mutant animal. Analysis of this region of the duct reveals that two inner hair cells are identifiable even at this early stage in apical development. E. In situ hybridization for Brn 3.1 in a cross section of the basal turn of the cochlear duct from an E17.5 control cochlea (ICR strain). A single inner hair cell (arrowhead) and three outer hair cells (arrows) express Brn 3.1. F, In situ hybridization for Brn 3.1 in a cross section of the basal turn of the cochlear duct from an E17.5 Jag2 mutant cochlea. Two inner hair cells (arrowhead) and three outer hair cells (arrows) express Brn 3.1. G. In situ hybridization for $M$ ath 1 in a cross section of the basal turn of the cochlear duct from an E17.5 wildtype cochlea. A single inner hair cell (arrowhead) and three outer hair cells (arrows) express Math1. This image is a composite of two photographs of the same section, taken at slightly different planes of focus. $\mathbf{H}$ In situ hybridization for Math1 in a cross section of the basal turn of the cochlear duct from an E17.5 Jag2 ${ }^{\Delta \mathrm{DSL}}$ cochlea. Two inner hair cells (arrowhead) and three outer hair cells (arrows) express Math1. All scale bars equal $25 \mu \mathrm{m}$. 


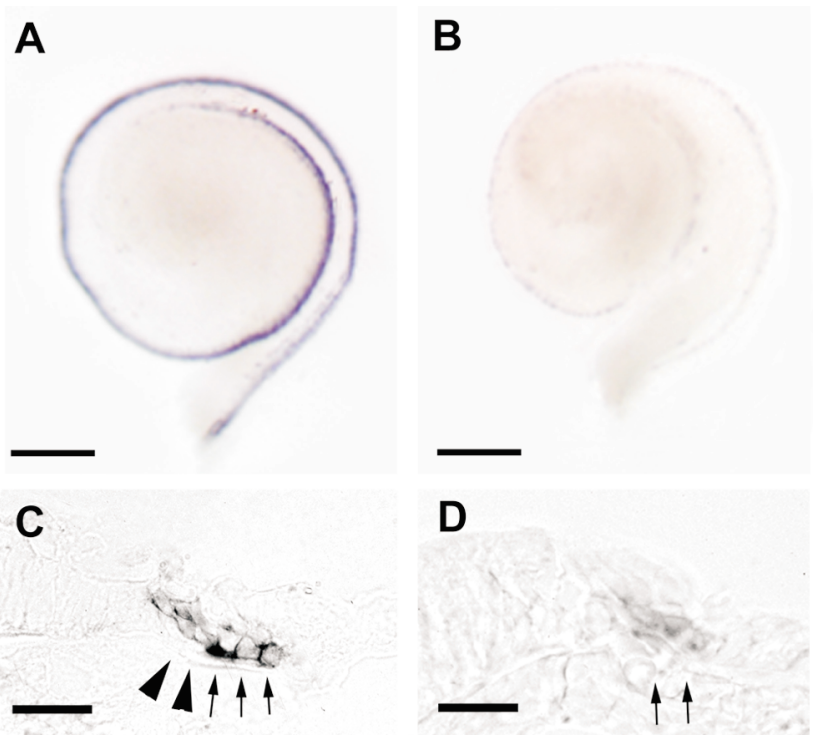

FIG. 5. Effects of deletion of Jag2 on the expression of HES5 in the developing organ of Corti. (A, B). Whole-mount in situ hybridization for HES5 in wildtype (A) vs. Jag2 mutant (B) cochleae at E17. In mutant cochleae, the expression of HES5 is significantly reduced and appears diffuse. Scale bars equal $250 \mu \mathrm{m}$. C. Cross section of the basal turn of the cochlear duct from an E17.5 wildtype cochlea. Expression of HES5 is restricted to cells located in the lower (nonhair cell) region of the developing organ of Corti. Arrows indicate three D eiter's cells; arrowheads indicate developing inner phalangeal cells. Scale bar equals $20 \mu \mathrm{m}$. D. Cross section of the basal turn of the cochlear duct from an E17.5 Jag2 mutant cochlea. Expression of HES5 is dramatically reduced in the sensory epithelium. Scale bar equal $20 \mu \mathrm{m}$.

the deletion of Jag2 results in a significant reduction in the activation of the Notch pathway in the developing organ of Corti.

\section{DISCUSSION}

Functional roles of proneural and neurogenic genes during cochlear development

Previous studies have demonstrated that proneural genes such as the Drosophila gene ato are initially expressed in a group of equivalent progenitor cells within the developing embryo but become progressively restricted to specific neural cell types (Akazawa et al. 1995; Jarmen et al. 1993, 1994; Ben-Arie et al. 1997; Gupta and Rodrigues 1997; Kim et al. 1997; Helms and Johnson 1998). Similarly, the expression of $M$ ath 1 in the mammalian ner vous system is relatively broad initial ly but ultimately becomes restricted to subtypes of neurons in the dorsal neural tube and cerebellum (Ben-Arie et al. 1997; H elms and Johnson 1998). The results of the present study and others (Lanford et al. 1999; Bermingham et al. 1999) indicate that a similar restriction occurs in the developing cochlear
E13
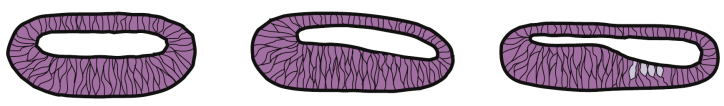

MATH1
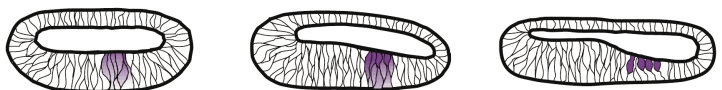

Jag2

DII1
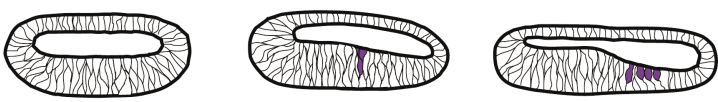

HES5
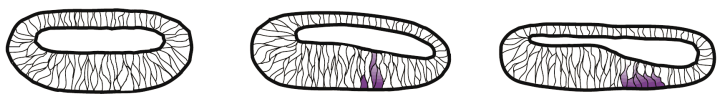

FIG. 6. Expression of proneural and neurogenic genes in the developing cochlea. Summary diagram shows the timing and relative positions of N otch1, M ath1, Jag2, DII1, and HES5 in cross sections through the base of the cochlea at E13, E15, and E17. At E13, Notch1 is expressed throughout the cochlear duct, while Math1 is expressed only in a subset of cells within the Notch1 domain. Transcripts for Jag2, DII1, and HES5 are not present in the epithelium at this time frame. Transcripts for the two ligands (Jag2 and DIII) begin to be expressed at E14.5 in a small subset of cells that appears to be located within the Math1 expression domain (not shown). Transcripts for Jag2 and DII1 continue to be present in the cochlear duct at E15, when HES5 expression begins. Presumably, the expression of HES5 is as a result of ligand-dependent N otch1 activation. Finally, by E17, M ath1, Jag2, and DII1 are localized to hair cells, while Notch1 and HES5 are expressed in nonsensory cells.

duct. Specifically, the number of cells that maintain $M$ ath 1 expression and differentiate as hair cells is greater in Jag2 mutant cochleae than in wildtype, suggesting that Jag2 plays a role in limiting the number of cells that differentiate as hair cells. While this result does not provide a direct link between Jag2 and the inhibition of M ath1, the data are consistent with previousstudies demonstrating that Jag2 activates the N otch pathway (Shawber et al. 1996; Jiang et al. 1998) and that activation of the pathway results in the repression of proneural genes and the restriction of progenitor cells to a particular fate (Anderson and Jan 1997). It is also possible that the deletion of Jag2 alters the expression of $M$ ath1 via another mechanism, however, such a mechanism has not been demonstrated.

Results from the present study also suggest that transcription of HES5 in the organ of Corti may be activated via Jag2- N otch binding. First, the spatiotemporal distribution of HES5 transcripts is consistent with the hypothesis that binding of Jag2 leads to activation of Notch and expression of HES5. Expression of HES5 begins approximately 24 hours after the onset of Jag2 expression in the cochlear duct, and transcripts for HES5 are distributed in a relatively broad pattern that appears to encompass the Jag2 expression domain. Second, the deletion of Jag2 results in a dramatic downregulation of HES5. This downregulation is consistent 
with previous studies demonstrating that the transcription of HES genes is dependent upon Notch activation ( Sasai et al. 1992; Jarriault et al. 1995, 1998; H sieh et al. 1997; Nishimura et al. 1998; O htsuka et al. 1999). Low levels of HES5 expression are present in Jag2 mutant cochleae; however, it is possible that this residual expression may be the result of activation via a second ligand, (e.g., Delta1; Morrison et al. 1999), or the activation of HES5 via proneural genes (Singson et al. 1994; Nellesen et al. 1999). This low level of activity may be responsible for the maintained development of supporting cells in Jag2 mutant cochleae ( Lanford et al. 1999).

The manner in which the inhibitory proteins encoded by HES5 interact with Math1 is uncertain. A specific molecular relationship between HES5 and $M$ ath1 has not been demonstrated. H owever, Akazawa at al. (1995) presented evidence that indicates that the bH LH proteins encoded by M ath 1 mediate E-boxdependent transcription through binding with a second bHLH protein, E47. That study also showed that the transcriptional activity of Math1 proteins is completely antagonized by the presence of bH LH proteins encoded by another HES gene, HES1 (Tomita et al. 1996; O htsuka et al. 1999). In a separate study, HES5 proteinswere shown to al so form nonfunctional heterodimers with E47 and to repress E47-induced transcription (Akazawa et al. 1992). Consequently, expression of HES5 proteins may downregulate the activity of Math1 proteins by competitively binding with E47.

Interestingly, the complementary patterns of HES5 vs. Mathl expression in the later stages of cochlear development (e.g., E17) suggest that HES5 may also regulate the transcription of the $M$ ath 1 gene itself. DNA footprinting analysis has shown that HES5 proteins bind to the CACNAG consensus sequence ( $N$ box) and, therefore, may be capable of repressing transcription directly (Akazawa et al. 1995). H owever, it is not known whether the $M$ ath 1 promoter contains an $\mathrm{N}$-box, or whether binding of HES5 is sufficient to downregulate the expression of $M$ ath1. Consequently, the function of HES5 as are pressor of M ath1 transcription remains speculative.

Role of proneural and neurogenic genes in the development of the organ of Corti; summary and model

Figure 6 provides a summary of neurogenic and proneural gene expression in the developing cochlea based on the results presented here and in a number of other studies. Notch 1 is expressed throughout the cochlear duct during the time frame in which cochlear progenitor cells become committed to sensory vs. nonsensory cell fates (E12-E17) (Lindsell et al. 1996;
Lewis et al. 1998; Lanford et al. 1999). Beginning on E13, transcripts for $M$ ath1 can be detected within the ventral floor of the cochlear duct, in a band of expression near the center of the epithelium. Analysis of later time points $(E 15, E 17)$ indicates that the band of expression of $M$ ath1 correlates with the developing sensory region of the duct. Beginning on E14, transcripts for Jag2 and DII1 can be detected in the base of the cochlea in a band of cells only 1-2 cell diameters in width (Lanford et al. 1999; Morrison et al. 1999). The position of these cells within the epithelium appears to be coincident with the neural edge of the $M$ ath 1 expression domain. Expression of these ligands is followed closely by the expression of HES5 transcripts at E15, which also appears to be coincident with the domain of $M$ ath 1 expression. In addition, the HES5 expression band appears to be subdivided by interceding HES5-negative cells. Finally, as the sensory epithelium matures through E17, transcripts for Jag2, DII1, and $M$ ath 1 are localized to hair cells within the organ of Corti, while transcripts for Notch1 and HES5 are restricted to supporting cells.

Based on the combined results of this and other studies, we propose the following working model for the molecular interactions that play a role in the development of the cochlear mosaic. Initially, as a result of the expression of $M$ ath1, a subset of cells within the cochlear duct become competent to develop as hair cells. Next, a subset of these cells begin to upregulate expression of the Notch ligands, Jag2 and DII1 (Jag2/ DII1). Expression of these ligands results in the activation of Notch in adjacent cells and the upregulation of HES5. Ultimately, expression of HES5 leads to the repression of $M$ ath1 and the diversion of progenitors from the hair cell fate.

The mechanisms that regulate the expression of many of these genes within individual cells are still uncertain. In particular, it is not clear how the expression of Jag2/DII1 is restricted to a subset of $M$ ath1positive progenitor cells. One possible explanation might be that expression of these ligands is dependent upon a critical level of $M$ athl. Therefore, as the level of $M$ ath 1 increases within a single cell, that cell would become competent to express Jag2/DII1. Since the expression of $M$ ath 1 occurs in a gradient along the basal-to-apical axis (and perhaps along the neural-toabneural axis as well) the number of cells that become competent to express Jag2/DII1 at any given moment in time will be extremely limited. In addition, once an individual cell becomes competent to express these ligands, that cell would then activate $\mathrm{N}$ otch in its immediate neighbors, thus greatly reducing the number of cells that ultimately express Jag2/ DII1.

Clearly, further studies are necessary to clarify the 
role of $M$ ath1 in the development of the sensory epithelium, as well as additional aspects of Notch signaling in this system. For example, while deletion of Jag2 results in the downregulation of HES5 expression and an increased production of hair cellsin the epithelium, it is not clear that expression of HES5 alone would be sufficient to eliminate the overproduction of hair cells. In addition, deletion of Jag2 doesnot result in a significant disruption of the cellular pattern within the organ of Corti, suggesting that other factors contribute to the formation of the cellular mosaic. Clearly, the roles of other Notch ligands, such as DII1, and other HESrelated genes, such as HES1 and the recently cloned H ey1, H ESr1, and H ey2 (Tomita et al. 1996; Kokubo et al. 1999; Leimeister et al. 1999), should be investigated to determine the role that these factors might play in Notch signaling during cochlear development. In addition, other factorsunrelated to the N otch path way probably play a role in the specification of cell types in the sensory epithelium.

\section{ACKN O W LED G MENTS}

The authors wish to thank Maura Donohue and Leanne Sleer for excellent technical assistance and R. Kageyama for kindly providing CDNAs. PJL was supported by an NRSA from NIDCD. This research was supported by grants from the M arch of Dimes (TG and MWK), the NINDS (NS36437) (TG), the National Organization for Hearing Research (MWK), and an Independent Scientist Award (K02) from NIDCD (MWK).

\section{REFEREN CES}

Adam J, Myat A, Le Roux I, Eddison M, Henrique D, Ish-H orowicz $D$, LEWIS J. Cell fate choices and the expression of Notch, Delta and Serrate homologs in the chick inner ear:parallels with Drosophila sense-organ development. Development 125:4645-4654, 1998.

Akazawa C, SASA Y, Nakanishi S, Kageyama R. Molecular characterization of a rat negative regulator with a basic helix-loop-helix structure predominantly expressed in the developing nervous system. J. Biol. Chem. 267:21879-21885, 1992.

akazawa C, Ishibashi M, Shimizu C, Nakanishi S, Kageyama R. A mammalian helix-loop-helix factor structurally related to the product of Drosophila proneural gene atonal is a positive transcriptional regulator expressed in the developing nervous system. J. Biol. Chem. 270:8730-8738, 1995.

ANDERSON DJ, JAN YN The determination of the neuronal phenotype. CowAN WM, JeSSELL TM, ZIPURSKY SL. Molecular and cellular approaches to neural development Oxford University Press New York, 1997, 26-63.

Artavanis-Tsakonas S, Matsuno K, Fortini ME. Notch signaling. Science 268:225-268, 1995.

Ben-Arie N, Bellen HJ, Armstrong DL, McCall aE, Gordadze PR, GUO Q , MATZUK M M , ZOGH BI HY. M ath1 is essential for genesis of cerebellar granule neurons. Nature 390:169-172, 1997.

Bermingham NA, Hassan BA, Price SD, Vollrath MA, Ben-Arie N, Eatock RA, Bellen HJ, LySAKowski A, Zogh bI HY. M ath1: An essential gene for the generation of inner ear hair cells. Science 284:1837-1841, 1999.

BRAYSJ. Expression and function of enhancer of split bH LH proteins during Drosophila neurogenesis. Perspect. Dev. Neurobiol. 4:313323, 1997.

CAMPOS-ORTEGA JA. Genetic mechanisms of early neurogenesis in Drosophila melanogaster. Mol. Neurobiol. 10:75-89, 1995.

Corwin JT, Jones JE, Katayama A, Kelley MW, Warchol ME. Hair cell regeneration: the identities of progenitor cells, potential triggers and instructive cues. Ciba Found. Symp. 160:103-120, 1991.

Doe CQ, Goodman CS. Early events in insect neurogenesis: II. The role of cell interactions and cell lineage in the determination of neuronal precursor cells. Dev. Biol. 111:206-219, 1985.

DORSKY RI, ChANG WS, RAPAPORT DH, HARRIS WA. Regulation of neuronal diversity in the $X$ enopus retina by delta signaling. Nature 385:67-70, 1997.

ERKman L, McEvilly RJ, Luo L, Ryan AK, HoOshmand F, O'Connell SM, Keithley EM, Rapaport DH, Ryan AF, RosenFELD MG. Role of transcription factors Brn-3.1 and Brn-3.2 in auditory and visual system development. Nature 381:603-606, 1996.

Giebel B, Campos-O rtega JA. Functional dissection of the Drosophila enhancer of split protein, a suppressor of neurogenesis. Proc. Natl. Acad. Sci. USA. 94:6250-6254, 1997.

GUPTA BP, RodRIGUES V. A tonal is a proneural gene for a subset of olfactory sense organs in Drosophila. Genes Cells 2:225-233, 1997.

HADDON C, JIANG YJ, SMITHERS L, LeWIS J. Delta-Notch signaling and the patterning of sensory cell differentiation in the zebrafish ear: evidence from the mind bomb mutant. Development 125:46374644, 1998.

Hasson T, Heintzelman MB, Santos-Sacchi J, Corey DP, MooseKER MS. Expression in cochlea and retina of myosin VIIa, the gene product defective in U sher syndrome type 1B. Proc. Nat. Acad. Sci. USA. 92:9815-9819, 1995.

Hasson T, Gillespie PG, Garci JA, MacDonald RB, Zhao Y, Yee AG, MOOSEKER MS, COREY DP. Unconventional myosins in innerear sensory epithelia. J. Cell Biol. 137:1278-1307, 1997.

Helms AW, Johnson JE. Progenitors of dorsal commissural interneurons are defined by $M$ ath 1 expression. Development 125:919-928, 1998.

H Sieh JJ, NofZiger DE, Weinmaster G, Hayward SD. Epstein-Barr virus immortalization: Notch2 interacts with CBF1 and blocks differentiation. J. Virol. 71:1938-1945, 1997.

Ishibashi M, Ang SL, Shiota K, Nakanishi S, Kageyama R, GuilleMOT F. Targeted disruption of mammalian hairy and enhancer of split homolog 1 (HES1) leads to up-regulation of neural helixloop-helix factors, premature neurogenesis, and severe neural tube defects. Genes Dev. 9:3136-3148, 1995.

JARMAN AP, GRAU Y, JAN LY, JAN YN. A tonal is a proneural gene that directs chordotonal organ formation in the Drosophila peripheral ner vous system. Cell. 73:1307-1321, 1993.

JaRman AP, GReLl EH, Ackerman L, Jan LY, Jan YN. A tonal is the proneural gene for Drosophila photoreceptors. Nature 369:398400, 1994.

Jarriault S, Brou C, Logeat F, Schroeter EH, Kopan R, Israel A. Signaling downstream of activated mammalian Notch. Nature 377:288-289, 1995.

Jarriault S, Le Bail O, hirsinger E, Pourquie O, Logeat F, Strong CF, Brou C, Seidah NG, Israel A. Deltal activation of Notch-1 signaling results in $\mathrm{HES1}$ transactivation. Mol. Cell. Biol. 18:7423-7431, 1998.

Jiang R, Lan Y, Chapman HD, Shawber C, Norton CR, Serreze DV, Weinmaster G, GridLey T. Defects in limb, craniofacial and thymic development in Jagged2 mutant mice. Genes Dev. 12:10461057, 1998. 
JIMENEZ G, ISH-H OROWICZ D. A chimeric enhancer-of-split transcriptional activator drives neural development and acheatescuteexpression. Mol. Cell. Biol. 17:4355-4362, 1997.

KAGEYAMA R, NAKANISHI S. Helix-loop-helix factors in growth and differentiation of the vertebrate nervous system. Curr. Opin. Genet. Dev. 7:659-665, 1997.

Kaufman MH. The Atlas of Mouse Development. Academic Press London, 1992, 123-370.

Kelley MW, Talreja DR, Corwin JT. Replacement of hair cells after laser microbeam irradiation in cultured organs of corti from embryonic and neonatal mice. J. Neurosci. 15:3013-3026, 1995.

KIM P, HeLMS AW, JOHNSON JE, ZIMmERMAN K. XATH 1, a vertebrate homolog of Drosophila atonal, induces neuronal differentiation within ectodermal progenitors. Dev. Biol. 187:1-12, 1997. DOI: 10.1006/ dbio.1997.8572.

KOKUBO H, LUN Y, JOHNSON RL. Identification and expression of a novel family of bHLH CDNAs related to Drosophila hairy and Enhancer of split. Biochem. Biophys. Res. Commun. 260:459-465, 1999. DOI: $10.1006 /$ bbrc.1999.0880.

Lanford PJ, LAN Y, JIANG R, LiNDSELL C, Weinmaster G, GRIDLEY T, KELLEY MW. Notch signaling pathway mediates hair cell development in mammalian cochlea. Nat. Gen. 21:289-292, 1999.

Leimeister C, Externbrink A, KLamt B, Gessler M. H ey genes: a novel subfamily of hairy-and Enhancer of split related genes specifically expressed during mouse embryogenesis. Mech. Dev. 85:173177, 1999. DOI: 10.1016/ S0925-4773 (99) 00080-5.

LEWIS J. Rules for the production of sensory cells. Ciba Found. Symp. 160:25-39, 1991.

LEWIS J. Neurogenic genes and and vertebrate neurogenesis. Curr. Opin. Neurobiol. 6:3-10, 1996.

Lewis aK, Franz GD, Carpenter Da, de Sauvage FJ, Gao W-Q. Distinct expression patterns of $N$ otch family receptors and ligands during development of the mammalian inner ear. Mech. Dev. 78:159-163, 1998. DOI: 10.1016/ S0925-4773 (98) 00165-8.

LIM DJ, ANNiko M. Developmental morphology of the mouse inner ear: A scanning electron microscopic obser vation. Acta O tolaryngol. Suppl. 422:1-69, 1985

LIM DJ, Rueda J. Structural development of the cochlea. RomAnd R. Development of auditory and vestibular systems 2. Elsevier $\mathrm{New}$ York, 1992, 33-58.

Lindsell C, Boulter J, Disibio G, Gossler A, Weinmaster G. Expression patterns of Jagged, Delta1, N otch1, N otch2 and Notch3 genes identify ligand-receptor pairs that may function in neural development. Mol. Cell. Neurosci. 8:14-27, 1996. DOI: 10.1006/ mone.1996.0040.

Luo B, Aster CJ, Hasserjian RP, Kuo F, SkLar J. Isolation and functional analysis of a cDNA for human Jagged2, a gene encoding a ligand for the $\mathrm{N} 1$ receptor. Mol. Cell. Biol. 17:6057-6067, 1997.

Ma Q, Kintner C, Anderson DJ. Identification of neurogenin, a vertebrate neuronal determination gene. Cell. 87:43-52, 1996.

ModelLeL J. Patterning of the adult peripheral nervous system of Drosophila. Perspect. Dev. Neurobiol. 4:285-296, 1997.

Morrison A, Hodgetts C, Gossler A, LeWIS J. Expression of Delta1 and Serratel (Jagl) in the mouse inner ear. Mech. Dev. 84:169-172, 1999. DOI: 10.1016/ S0925-4773 (99) 00066-0.

MUSKAVITCH MA. Delta-Notch signaling and Drosophila cell fate choice. Dev. Biol. 166:415-430, 1999. DOI: 10.1006/ dbio. 1994.1326.

Nakao K, Campos-Ortega JA. Persistent expression of genes of the enhancer of split complex suppresses neural development in Drosophila. Neuron. 16:275-286, 1996.

NeLLesen DT, LAI EC, POSAKONY JW. Discrete enhancer elements mediate selective responsiveness of enhancer of split complex genes to common transcriptional activators. Dev. Biol. 213:33-53, 1999. DOI: 10.1006/ dbio.1999.9324.

NISHIMURA S, ISAKA F, ISHIBASHI M, TOMITA K, TSUdA H, NAKANISHI S, KageYAMA R. Structure, chromosomal locus and promoter of mouse HES2 gene, a homolog of Drosophila hairy and enhancer of split. Genomics 49:69-75, 1998. DOI: 10.1006/ geno.1998.5213.

OhtSuka T, Ishibashi M, Gradwohl G, NakanishI S, Guillemot F, KAGEYAMA R. HES1 and HES5 as Notch effectors in mammalian neuronal differentiation. EMBO J. 18:2196-2207, 1999.

Pompa Jl de la, Wakeham A, Correia KM, Samper E, Brown $S$, Aguilera RJ, Nakano T, Honjo T, Mak TW, Rossant J, Conlon RA. Conservation of the Notch signaling pathway in mammalian neurogenesis. Development. 124:1139-1148, 1997.

Sasai Y, Kageyama R, Tagawa Y, Shigemoto R, Nakanishi S. Two mammalian helix-loop-helix factors structurally related to Drosophila hairy and enhancer of split. Genes Dev. 6:2620-2634, 1992.

Shailam R, Lanford PJ, Dolinsky CM, Norton CR, Gridley T, KELLEY MW. Expression of proneural and neurogenic genes in the embryonic mammalian vestibular system. J. Neurocytol. 28:809-819, 1999.

Shawber C, Boulter J, Lindsell CE, Weinmaster G. Jagged2: a serratelike gene expressed during rate embryogenesis. Dev. Biol. 180:370-376, 1996. DOI: 10.1006/ dbio.1996.0310.

Singson A, Leviten MW, Bang AG, Hua XH, Posakony JW. Direct downstream targets of proneural activators in the imaginal disc include genes involved in lateral inhibitory signaling. Genes Dev. 8:2058-2071, 1999.

SO MMER L, MA Q , ANDERSON DJ. N eurogenins, a novel family of atonalrelated bH LH transcription factors, are putative mammalian neuronal determination genes that reveal progenitor cell heterogeneity in the developing CNS and PNS. Mol. Cell. Neurosci. 8:221-241, 1996. DOI: 10.1006/ mcne.1996.0060.

Tomita K, Ishibashi M, NaKahara K, Ang SL, Nakanishi S, GuilleMOT F, KAGEYAMA R. Mammalian hairy and enhancer of split homolog 1 regulates differentiation of retinal neurons and is essential for eye morphogenesis. Neuron 16:723-734, 1996.

WoLfF T, READY DF. Pattern formation in the Drosophila retina. BATE M, MARTINEZ A. The development of D rosophila melanogaster Cold Spring Harbor Laboratory Press Cold Spring H arbor, NY, 1993.

WIKINSON DG, NEITO MA. Detection of messenger RNA by in situ hybridization to tissue sectionsand whole mounts. Meth. Enzymol. 225:361-372, 1993.

Xiang M, Gan L, LI D, Chen ZY, Zhou L, O'Malley BW, KLein JR WH, NATHANS J. Essential role of POU-domain factor Brn-3c in auditory and vestibular hair cell development. Proc. Natl. Acad. Sci. USA. 94:9445-9450, 1997a. 